\title{
Disruption of pair bonding induces pregnancy failure in newly mated Mongolian gerbils (Meriones unguiculatus)
}

\author{
M. L. Norris \\ AFRC Institute of Animal Physiology, Animal Research Station, 307 Huntingdon Road, \\ Cambridge CB3 OJQ, U.K.
}

\begin{abstract}
Summary. The efficacy of several exteroceptive factors including exposure to a 'strange' male, were tested to induce pregnancy failure in newly mated Mongolian gerbils. Only physical separation of monogamous pairs consistently induced pregnancy failure. This phenomenon was restricted to the immediate period after mating and is believed to be due to disruption of pair bonding, with associated luteal and implantation failure. Concurrent lactation annulled this effect.
\end{abstract}

\section{Introduction}

Various exteroceptive factors are able to interfere with fertility in different mammals with the best documented one being that of the 'strange' male effect in the laboratory mouse originally noted by Bruce $(1959,1960 \mathrm{a}, \mathrm{b})$, and demonstrated in several other rodent species (see Marchlewska-Koj, 1983 ) but not in the rat (Davis \& de Groot, 1969) or rabbit (Bell \& Reece, 1983). Although a reduced level of fertility observed in newly mated Mongolian gerbils was attributed to stressful exteroceptive factors at critical times in pregnancy, the 'strange' male effect was not detected (Norris \& Adams, 1979). However, a report of a high degree of susceptibility to pregnancy block in gerbils induced by a 'strange' male or female (Rohrbach, 1982) prompted the present investigation to identify the exteroceptive factor(s) apparent in our earlier study (Norris \& Adams, 1979), and re-examine for the existence of the 'strange' male effect.

\section{Materials and Methods}

A total of 260 female Mongolian gerbils from the ARS colony maintained under conditions described elsewhere (Norris \& Adams, 1981) were paired at 60 days of age with older proven males ( = stud males). After the birth of a litter, vaginal smears were taken by lavage between 09:00 and 10:00 h. After confirmation of mating (Day 1 post coitum) 20 females were allocated to Exp. 11, and litters were removed from 240 females before they were allocated, 20 per group, to the other 12 experiments (see Tables $1 \& 2$ ). The mean age of females at mating was $104 \pm 1$ days. The females in Exp. 1 were left undisturbed and those in Exp. 2 had additional vaginal smears taken daily between 09:00 and 10:00 h on Days 2-6. Experiments 6 and 7 consisted of females from albino pairs, and agouti 'strange' males were used in Exp. 7.

Experiments 1-9 were designed to test the effect of the following 8 exteroceptive factors: (a) vaginal smears taken on Days 2-6; (b) female and stud male moved to fresh box on Day 1; (c) female and stud male returned to original box on Day 9; (d) female moved to fresh box on Day 1; (e) female returned to original box containing stud male on Day 9; (f) 'strange' male; (g) female returned to original box on Day 9; (h) litter and stud male removed on Day 1. The distribution of these factors within the 9 experiments is given in Table 1 . All females were checked daily for littering from Day 20. 
The effect on implantation of the time after copulation when the female was removed from her stud male was examined in Exp. 10. Experiment 11 tested the effect of concurrent lactation on females separated from their partners on Day 1, and Exps 12 and 13 examined for early (preimplantation) effects after separation of newly mated females. Details of these experiments are presented in Table 2. At autopsy, the ovaries of all females were examined for corpora lutea (CL). After separation from their stud males on Day 1, the females in Exp. 10 were examined for implantations on Day 10 ( 2 days after implantation). The lactating females in Exp. 11 had implantation induced by litter removal on Day 8 and were examined for implantations 4 days later. The females in Exps 12 and 13 were examined on Day 6, when embryos would be expected to be in the uterus. The oviducts and uteri were dissected and flushed with phosphate-buffered saline (pH 7.4) and the flushings were searched for embryos using a stereomicroscope. Fisher's exact test for contingency tables was used to examine differences in the proportions of females littering in the various experiments.

\section{Results}

In Exp. 2 the cellular content of vaginal smears taken Days 2-6 revealed little change, leucocytes and epithelial cells being dominant, and no instances of cornification and/or spermatozoa were noted. The intervals from mating to littering fell into 2 distinct groups, 24-26 days $(\mathrm{N}=15)$ and 40-43 days $(\mathrm{N}=5)$; the former group represented the reported gestation length for this species (Nakai, Nimura, Tamura, Shimizu \& Nishimura, 1960; Marston \& Chang, 1965), and was used to determine fertility in all experiments assessed at term (Table 1). Similar levels of fertility were found in Exps 1-4, indicating that factors (a), (b) and (c) had no significant effect. A significantly reduced level of fertility was found in Exps 5-9, with factor (d) implicated in Exps 5-8 and factor (h) in Exp. 9 (see Table 1). Both of these factors result in physical separation of the established pair. In Exp. 7 all of the young born to the 7 albino females lacked eye pigmentation, thereby confirming that these litters resulted from the observed post-partum matings with albino stud males.

In each of Exps 10 and 11 there were 14 females with CL of normal appearance and implantations present at autopsy on Days 10 and 12 respectively (Table 2). In Exp. 12 only 3 females had CL present and embryos of normal appearance were recovered from the uteri on Day 6 . Of the 17 females with failed pregnancies, 11 were without $C L$ and 6 showed advanced CL regression.

Table 1. The effect of exteroceptive factors on the littering of uniparous Mongolian gerbils mated post partum

\begin{tabular}{|c|c|c|c|c|}
\hline \multirow[b]{2}{*}{ Exp. } & \multirow{2}{*}{$\begin{array}{l}\text { Factors } \\
\text { tested* }\end{array}$} & \multicolumn{2}{|c|}{ Animal movements } & \multirow{2}{*}{$\begin{array}{l}\text { No. of females } \\
\text { littering }(\%)\end{array}$} \\
\hline & & Day 1 & Day 9 & \\
\hline I & Control & - & $\ldots$ & $18(90)$ \\
\hline 2 & (a) & - & - & $15(75)$ \\
\hline 3 & (b) & $q+$ stud $\sigma$ into fresh box & - & $19(95)$ \\
\hline 4 & (b) + (c) & $q+$ stud $\sigma^{*}$ into fresh box & $q+$ stud $\delta$ returned to original box & $16(80)$ \\
\hline 5 & (d) & $q$ into fresh box & - & $0(0)$ \\
\hline 6 & (d) $+(\mathrm{e})$ & $q$ into fresh box & $q$ returned to original box + stud $\delta$ & $2(10)$ \\
\hline 7 & (d) + (f) $+(e)$ & $q$ into fresh box + strange $\delta$ & $q$ returned to original box + stud $\delta$ & $7(35)$ \\
\hline 8 & (d) $+(\mathrm{g})$ & $q$ into fresh box & $\Phi$ returned to original box & $1(5)$ \\
\hline 9 & (h) & litter + stud $\partial \hat{\text { removed }}$ & - & $3(15)$ \\
\hline
\end{tabular}

*See 'Materials \& Methods'; 20 females/experiment.

Exps 1 vs 2, N.S.; Exps 1 vs 9, $P<0.001$; Exps 3 vs 5, $P<0.001$; Exps 4 vs 6, $P<0.001$; Exps 4 vs $7, P<0.01$; Exps 4 vs $8, P<0.001$; Exps 5 vs 9 , N.S.; Exps 6 vs 7, N.S. 
Table 2. The influence of the stud male and litter on the maintenance of pregnancy in uniparous Mongolian gerbils mated post partum

\begin{tabular}{|c|c|c|c|}
\hline Exp.* & Animal movements & $\begin{array}{l}\text { Day (p.c.) } \\
\text { of autopsy }\end{array}$ & $\begin{array}{c}\text { No. of } \% q \\
\text { pregnant }(\%)\end{array}$ \\
\hline 10 & $\begin{array}{l}\text { Litter removed on Day } 1 \\
\text { Q into fresh box on Day } 5\end{array}$ & 10 & $14(70)$ \\
\hline 11 & $\begin{array}{l}q+\text { litter into fresh box on Day } 1 \text {, } \\
\text { litter removed on Day } 8\end{array}$ & 12 & $14(70)$ \\
\hline 12 & $q$ into fresh box on Day 1 & 6 & $3(15)$ \\
\hline 13 & Litter and stud ${ }^{*}$ removed on Day 1 & 6 & $11(55)$ \\
\hline
\end{tabular}

*There were 20 females/experiment.

Exps 12 vs $13, P<0.05$.

Embryos were recovered from the oviducts of 4 females, from the oviducts and uteri of 4 females, from the uteri of 4 females, and 5 were without embryos. Most of the embryos were at various stages of degeneration. In Exp. 13, 5 of the 9 females with failed pregnancies had complete CL regression, 2 showed advanced regression and 2 had $\mathrm{CL}$ of normal appearance. Degenerating embryos were recovered from the oviducts of 3 females, from the oviducts and uteri of 3 females, from the uteri of 1 female, and 2 females were without embryos.

\section{Discussion}

Unlike the deermouse (Eleftheriou, Bronson \& Zarrow, 1962) and contrary to our earlier view for the gerbil (Norris \& Adams, 1979), potentially stressful exteroceptive factors such as handling, vaginal lavage (Exp. 2) and movement(s) of female + stud male (Exp 3 \& 4) had no adverse effects on fertility. However, the significant reduction in fertility apparent in Exps 5-9 is attributed to one common factor, separation of the newly mated female from her stud male. This factor was present in our earlier study (Norris \& Adams, 1979; Exp. 5) but its ability to influence fertility was not recognized. Exposure of the female to a 'strange' male over the entire preimplantation period did not result in increased pregnancy failure (Exps 6 \& 7), thereby confirming our earlier report (Norris \& Adams, 1979). Furthermore, the present result obtained using genetically colour-marked animals (Exp. 7), to identify any undetected matings by the strange male and thereby eliminate the occurrence of false positives following a pregnancy block, compares very favourably with our earlier result (Norris \& Adams, 1979; Exp. 3). The discrepancy between the present results and those of Rohrbach (1982) could be due to strain variation, or to differences in the methods used to assess fertility, i.e. littering in the present study, and vaginal smears by Rohrbach (1982). Difficulties have been noted concerning the reliability and interpretation of vaginal smears in the gerbil (Marston \& Chang, 1965; Barfield \& Beeman, 1968).

Although olfaction appears normally dominant in nocturnal species such as the gerbil (Thiessen, 1973), results obtained by Yahr (1977) suggest that, because the laboratory maintained gerbil is active night and day (Lerwill, 1974), visual and olfactory cues may be used, with the emphasis switching according to conditions. The present results suggesting that a more efficient block to pregnancy may be achieved by moving the female rather than the male from the established pairing box (Exps 5 vs 9, and Exps 12 vs 13) indicate the importance of olfaction, with the residual effect of the stud male being sufficient to influence some females after separation. This residual influence of the stud male was more marked when females were examined early in pregnancy, pregnancy block being detected in only $45 \%$ females on Day 6 (Exp. 13) compared with $85 \%$ failing to litter (Exp. 9). Gerbils maintained under laboratory and semi-natural conditions are monogamously pair forming and territorial, based on an olfactory discrimination via deposition of a pheromonal-containing sebum exudate from a specialized ventral gland (Marston \& Chang, 
1965; Thiessen, Owen \& Lindzey, 1971; Thiessen, 1973; Thiessen et al., 1974; Ågren, 1976, 1984). The obvious importance of olfaction facilitates individual recognition (Thiessen et al., 1974; Ågren, 1976; Halpin, 1976; Ågren \& Meyerson, 1977) and could form a functional social bonding mechanism via olfactory imprinting to promote familiarity and eliminate innate aggression. The present results would then stem from a sudden absence of familiar cues associated with the formerly bonded male. This interpretation agrees with earlier reports for voles and mice emphasizing the importance of the stud male in establishing and maintaining pregnancy (Richmond \& Stehn, 1976; Berger \& Negus, 1982; Yamazaki et al., 1983). Evidence for the vole (Milligan, 1979) suggests the existence of a system of rapid female imprinting at the time of mating, giving memory of the stud male. However, the present experiments with gerbils do not provide any information on the time of female exposure to the stud male required to enable putative imprinting and the establishment of pair bonding to occur. The significant pregnancy block achieved without female isolation (Exp. 7) accords with the view that the deleterious effects on fertility were induced by disruption of pair bonding. The fact that the 'strange' male appeared to act in a supportive fashion in raising the proportion of successful pregnancies by $25 \%$ (Exps 6 vs 7), could be due to visual cues in the absence of the more influential olfactory ones associated with the formerly bonded male.

The present results showed that the effect of disruption of pair bonding was: restricted to the immediate period after mating (Exp. 10); was annulled by concurrent lactation (Exp. 11); and was associated with $\mathrm{CL}$ failure, embryo degeneration and irregular transport (Exps 12 \& 13). These events are strikingly similar to those induced by 'strange' male urinary pheromones via olfaction in the mouse, thereby implying a neuroendocrine response to a specific stimulus resulting in cessation of luteotrophin (prolactin implicated) release from the anterior pituitary, followed by luteal failure, progesterone deficiency and pregnancy block (Bronson, 1971; Marchlewska-Koj, 1983). Supportive evidence for a 'pregnancy protective' effect from an assumed increase in circulating prolactin concentrations associated with lactation in the gerbil is that the presence of a litter significantly delayed CL regression, irrespective of whether the male was present or absent (Meckley \& Ginther, 1972 ), and a luteolytic effect of prostaglandin F-2 $\alpha$ was demonstrated in gerbils, but not in ones concurrently lactating (Chaichareon, Meckley \& Ginther, 1974).

It seems unwise to speculate on a possible biological role for disruption of pair bonding in view of our lack of knowledge regarding Mongolian gerbils in their natural environment, added to the fact that the laboratory gerbil is now highly inbred with a genetic variance approaching zero (Thiessen et al., 1971). As a general phenomenon pregnancy interruption remains to be conclusively demonstrated in the field, and its adaptive significance is still questionable (Heske \& Nelson, 1984). While the effect of disruption of pair bonding would not typically occur in the gerbil for which pregnancy concurrent with lactation is the norm, this phenomenon must obviously be considered when planning certain experimental procedures, and it does present a potentially useful model for studies relating to preimplantation failure.

I thank Dr D. E. Walters, AFRC Statistics Group, Department of Applied Biology, University of Cambridge, for carrying out the statistical analyses and Dr M. A. H. Surani for his interest and helpful comments during the preparation of this manuscript.

\section{References}

Agren, G. (1976) Social and territorial behaviour in the Mongolian gerbil (Meriones unguiculatus) under seminatural conditions. Biol. Behav. 1, 267-285.

Ågren, G. (1984) Pair formation in the Mongolian gerbil. Anim. Behav. 32, 528-535.

Ågren, G. \& Meyerson, B.J. (1977) Influence of gonadal hormones and social housing conditions on agon- istic, copulatory and socio-sexual behaviour in the Mongolian gerbil (Meriones unguiculatus). Behav. Proc. 2, 265-282.

Barfield, M.A. \& Beeman, E.A. (1968) The oestrous cycle in the Mongolian gerbil (Meriones unguiculatus). $J$. Reprod. Fert. 17, 247-251.

Bell, D.J. \& Reece, C. (1983) An investigation into the 
'Bruce Effect' in domestic rabbits. In Chemical Signals in Vertebrates. Vol. 3, pp. 339-342. Eds D. Muller-Schwarze \& R. M. Silverstein. Plenum Press, New York.

Berger, P.J. \& Negus, N.C. (1982) Stud male maintenance of pregnancy in Microtus montanus. J. Mammal. $63,148-151$.

Bronson, F.H. (1971) Rodent pheromones. Biol. Reprod. 4, 344-357.

Bruce, H.M. (1959) An exteroceptive block to pregnancy in the mouse. Nature, Lond. 184, 105.

Bruce, H.M. (1960a) A block to pregnancy in the mouse caused by proximity of strange males. $J$. Reprod. Fert. 1, 96-103.

Bruce, H.M. (1960b) Further observations on pregnancy block in mice caused by the proximity of strange males. J. Reprod. Fert. 1, 311-312.

Chaichareon, D.P., Meckley, P.E. \& Ginther, O.J. (1974) Effect of prostaglandin $F_{2 \alpha}$ on corpora lutea in guinea pigs and Mongolian gerbils. Am. J. vet. Res. 35, 685-687.

Davis, D.L. \& de Groot, J. (1969) Failure to demonstrate olfactory inhibition of pregnancy ('Bruce effect') in the rat. Anat. Rec. 148, 366.

Eleftheriou, B.E., Bronson, F.H. \& Zarrow, M.X. (1962) Interaction of olfactory and other environmental stimuli on implantation in the deer mouse. Science, N.Y. 137, 764 .

Halpin, Z.T. (1976) The role of individual recognition by odours in the Mongolian gerbil (Meriones unguiculatus). Behaviour 58, 117-130.

Heske, E.J. \& Nelson, R.J. (1984) Pregnancy interruption in Microtus ochrogaster: laboratory artifact or field phenomenon? Biol. Reprod. 31, 97-103.

Lerwill, C.J. (1974) Activity rhythms of golden hamsters (Mesocricetus auratus) and Mongolian gerbils (Meriones unguiculatus) by direct observation. $J$. Zool., Lond. 175, 520-523.

Marchlewska-Koj, A. (1983) Pregnancy blocking by pheromones. In Pheromones and Reproduction in Mammals, pp. 151-174. Ed. J. G. Vandenbergh. Academic Press, New York.

Marston, J.H. \& Chang, M.C. (1965) The breeding, management and reproductive physiology of the Mongolian gerbil .(Meriones unguiculatus). Lab. Anim. Care 15, 3448.
Meckley, P.E. \& Ginther, O.J. (1972) Effects of litter and male on corpora lutea of the postpartum Mongolian gerbil. J. Anim. Sci. 34, 297-301.

Milligan, S.R. (1979) Pregnancy blockage and the memory of the stud male in the vole (Microtus agrestis). J. Reprod. Fert. 57, 223-225.

Nakai, K., Nimura, M., Tamura, M., Shimizu, S. \& Nishimura, H. (1960) Reproduction and post natal development of the colony bred Meriones unguiculatus kurauchii (mori). Bull. Exp. Anim. (Japan) 9, $157-159$.

Norris, M.L. \& Adams, C.E. (1979) Exteroceptive factors and pregnancy block in the Mongolian gerbil, Meriones unguiculatus. J. Reprod. Fert. 57, 401-404.

Norris, M.L. \& Adams, C.E. (1981) Mating post partum and length of gestation in the Mongolian gerbil (Meriones unguiculatus). Lab. Anim. 15, 189-191.

Richmond, M. \& Stehn, R. (1976) Olfaction and reproductive behaviour in microtine rodents. In Mammalian Olfaction, Reproductive Processes and Behaviour, pp. 197-217. Ed. R. L. Doty. Academic Press, London.

Rohrbach, C. (1982) Investigation of the Bruce effect in the Mongolian gerbil (Meriones unguiculatus). $J$. Reprod. Fert. 65, $411-417$.

Thiessen, D.D. (1973) Footholds for survival. Am. Scient. 61, 346-351.

Thiessen, D.D., Owen, K. \& Lindzey, G. (1971) Mechanisms of territorial marking in the male and female Mongolian gerbil (Meriones unguiculatus). J. comp. Physiol. Psychol. 77, 38-47.

Thiessen, D.D., Regnier, F.E., Rice, M., Goodwin, M., Isaaks, N. \& Lawson, N. (1974) Identification of a ventral scent marking pheromone in the male Mongolian gerbil (Meriones unguiculatus). Science, N.Y. 184, 83-85.

Yahr, P. (1977) Social subordination and scent-marking in male Mongolian gerbils (Meriones unguiculatus). Anim. Behav. 25, 292-297.

Yamazaki, K., Beauchamp, G.K., Wysocki, C.J., Bard, J., Thomas, L. \& Boyse, E.A. (1983) Recognition of $\mathrm{H}-2$ types in relation to the blocking of pregnancy in mice. Science, N.Y. 221, 186-188.

Received 5 November 1984 